OPEN ACCESS

Edited by: Georg A. Feichtinger, Evotec GT, Austria

Reviewed by: Nihay Laham Karam, University of Eastern Finland, Finland

Shen Shen,

Biogen Idec (United States),

United States

${ }^{*}$ Correspondence:

Bas Blits

b.blits@nin.knaw.n

${ }^{\dagger}$ Present address: Bas Blits,

Sana-Gen, Amsterdam, Netherlands

Specialty section:

This article was submitted to Preclinical Cell and Gene Therapy,

a section of the journal

Frontiers in Bioengineering and

Biotechnology

Received: 11 March 2021

Accepted: 24 June 2021

Published: 03 August 2021

Citation:

Pietersz KL, Plessis FD,

Pouw SM, Liefhebber JM,

van Deventer SJ, Martens GJM, Konstantinova PS and Blits B (2021) PhP.B Enhanced Adeno-Associated Virus Mediated-Expression Following Systemic Delivery or Direct Brain Administration Front. Bioeng. Biotechnol. 9:679483. doi: 10.3389/fbioe.2021.679483

\section{PhP.B Enhanced Adeno-Associated Virus Mediated-Expression Following Systemic Delivery or Direct Brain Administration}

\author{
Kimberly L. Pietersz',2, Francois Du Plessis' ${ }^{1}$, Stephan M. Pouw'1, \\ Jolanda M. Liefhebber ${ }^{1}$, Sander J. van Deventer ${ }^{1,3}$, Gerard J. M. Martens ${ }^{2}$, \\ Pavlina S. Konstantinova ${ }^{1}$ and Bas Blits ${ }^{1 * t}$
}

\begin{abstract}
'Department of Research \& Development, uniQure Biopharma B.V., Amsterdam, Netherlands, ${ }^{2}$ Department of Molecular Animal Physiology, Faculty of Science, Centre for Neuroscience, Donders Institute for Brain, Cognition and Behavior, Radboud University, Nijmegen, Netherlands, ${ }^{3}$ Department of Gastroenterology and Hepatology, Leiden University Medical Center, Leiden, Netherlands
\end{abstract}

Of the adeno-associated viruses (AAVs), AAV9 is known for its capability to cross the blood-brain barrier (BBB) and can, therefore, be used as a noninvasive method to target the central nervous system. Furthermore, the addition of the peptide PhP.B to AAV9 increases its transduction across the BBB by 40 -fold. Another neurotropic serotype, AAV5, has been shown as a gene therapeutic delivery vehicle to ameliorate several neurodegenerative diseases in preclinical models, but its administration requires invasive surgery. In this study, AAV9-PhP.B and AAV5-PhP.B were designed and produced in an insect cell-based system. To AAV9, the PhP.B peptide TLAVPFK was added, whereas in AAV5-PhP.B (AQTLAVPFKAQAQ), with AQ-AQAQ sequences used to swap with the corresponding sequence of AAV5. The addition of PhP.B to AAV5 did not affect its capacity to cross the mouse BBB, while increased transduction of liver tissue was observed. Then, intravenous (IV) and intrastriatal (IStr) delivery of AAV9PhP.B and AAV5 were compared. For AAV9-PhP.B, similar transduction and expression levels were achieved in the striatum and cortex, irrespective of the delivery method used. IStr administration of AAV5 resulted in significantly higher amounts of vector DNA and therapeutic miRNA in the target regions such as striatum and cortex when compared with an IV administration of AAV9-PhP.B. These results illustrate the challenge in developing a vector that can be delivered noninvasively while achieving a transduction level similar to that of direct administration of AAV5. Thus, for therapeutic miRNA delivery with high local expression requirements, intraparenchymal delivery of AAV5 is preferred, whereas a humanized AAV9-PhP.B may be useful when widespread brain (and peripheral) transduction is needed.

Keywords: aav, intrastriatal, intravenous, CNS, PhP.B 


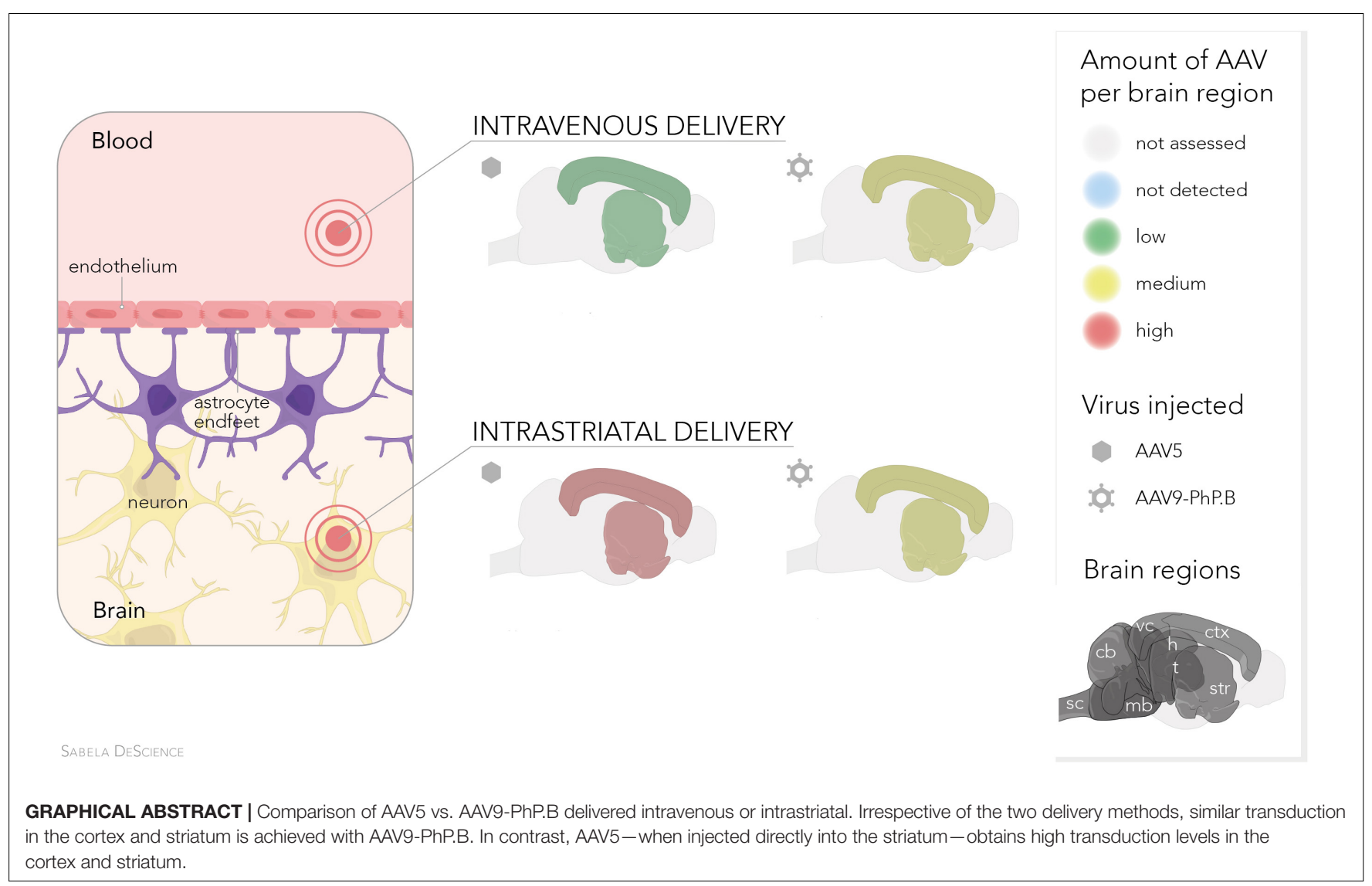

\section{INTRODUCTION}

Neurodegenerative diseases are a heterogeneous group of multisystem disorders affecting the central nervous system (CNS), and treatment options for those are limited. An important and challenging aspect of the treatment of these diseases is efficient drug target delivery. Viral vectors harboring therapeutic nucleic acid molecules have to be delivered to the CNS to accomplish their therapeutic action at the desired site. The delivery of viral vectors is a part of molecular neurosurgery, thereby treating disease on a molecular level. Various disease-modifying therapies are in the (pre)clinical stage for neurodegenerative diseases based on adeno-associated virus (AAV) as the delivery tool of nucleic acids (Chen et al., 2020; Mijanović et al., 2020).

One of the examples of such a preclinical program is for the treatment of Huntington's disease (HD). Intrastriatal (IStr) delivery of AAV carrying a huntingtin silencing miRNA resulted in a successful reduction in huntingtin mRNA and protein in the brains of mouse and minipig HD models (Evers et al., 2018; Spronck et al., 2019). The produced miRNA lowered cytoplasmic and nuclear gene expression and showed therapeutic spread through extracellular vesicles without off-target effects (Keskin et al., 2019). This miRNA technology has also been used as silencing strategies for Spinocerebellar Ataxia Type 3 (Martier et al., 2019b) and Amyotrophic Lateral Sclerosis (ALS) (Martier et al., 2019a).
The direct IStr administration of AAV has been used to supply the brain locally with the therapeutic doses of the miRNA (Evers et al., 2018; Spronck et al., 2019). However, this invasive intervention requires a well-equipped and trained neurosurgical team. Intravenous (IV) administration would be an attractive alternative since it is less invasive. Nevertheless, an AAV capsid that can cross the blood-brain barrier (BBB) is required when targeting the CNS by IV administration. AAV9 has been shown to have this capability, especially in neonates (Foust et al., 2008). The number of vector copies needed to reach therapeutic doses in the brain may lead to elevated aminotransferase levels, an indicator of liver damage (Mendell et al., 2017). For the probable cause of systemic adverse events, it is currently challenging to use IV administration of AAV9 for neurodegenerative diseases in adults.

Various attempts have been undertaken to improve the capability and efficiency of AAV to cross the BBB. A variant of AAV9, named AAV9-PhP.B, was reported to enhance brain tissue transduction, such as the cortex, by 40 -fold compared with its AAV9 ancestor (Deverman et al., 2016). The enhancement of AAV9 by PhP.B appears to occur only in rodents (Hordeaux et al., 2018), with a preference for the $\mathrm{C} 57 \mathrm{Bl} / 6$ strain, the strain in which PhP.B was developed and tested (Hordeaux et al., 2018). Studies in nonhuman primates (Liguore et al., 2019) and marmosets (Matsuzaki et al., 2018) have not shown any increase in the transduction levels of AAV9-PhP.B when compared to AAV9. PhP.B binds to the Ly6a receptor, a GPI (glycophosphatidylinositol)-anchored surface protein highly 
expressed in the microvascular endothelial cells of C57Bl/6 mice (Hordeaux et al., 2019; Huang et al., 2019). Unfortunately, Ly6a is absent in endothelial cells of primates (Hordeaux et al., 2019), which explains why no increase is observed in BBB crossing compared with AAV9. These results limit the use of PhP.Bcontaining vectors in humans. However, AAV9-PhP.B can still be used as a benchmark for preclinical studies in $\mathrm{C} 57 \mathrm{Bl} / 6$ mice. The knowledge gained from these studies could be used to rationally develop novel vectors that cross the BBB.

PhP.B is constructed by inserting a 7-mer peptide at position I-588. The insertion site is located outside of the capsid on the tip of a loop at the 3-fold axis of symmetry, facilitating interaction with the receptors of targeted cells. AAV9-PhP.B has shown to be functional in mouse models. For example, AAV9-PhP.B-based gene therapy has been shown to reduce $\alpha$-synuclein pathology in a preclinical mouse model (Morabito et al., 2017).

We hypothesized that the addition of PhP.B could also be used to enhance BBB crossing of other serotypes. Zhang et al. (2011) showed that the natural serotypes of AAV2 and AAV5 do not cross the BBB even in neonatal mice (Zhang et al., 2011). AAV5 is the most divergent of the naturally occurring AAVs. After local administration, AAV5 shows widespread transduction of the brain (Samaranch et al., 2017), making this vector a useful candidate to test the limits of PhP.B. This widespread targeting has been shown in a variety of animal models such as mice, rats, nonhuman primates, and minipigs (Emborg et al., 2014; Miniarikova et al., 2017; Evers et al., 2018; Caron et al., 2019). Furthermore, AAV5 has been shown to specifically target astrocytes and specialized neurons such as dopaminergic and motor neurons derived from the human-induced pluripotential stem cells (Martier et al., 2019a). In in addition to neuronal tropism AAV5 could be delivered systemically in a noninvasive manner would be ideal. Therefore, PhP.B was added to AAV5 to explore the enhanced crossing of the $\mathrm{BBB}$.

First, AAV5 and AAV9 were modified by inserting the 7mer PhP.B, and the vectors were produced in the baculovirus expression system. This system facilitates scaling up production (Kotin and Snyder, 2017). Then, these new capsids were evaluated in vitro with respect to the green fluorescent protein (GFP) expression and dose-response quantified by luciferase activity. The capability of capsids to cross the BBB of mice was assessed at a relatively low dose to remain below the saturation threshold. Finally, IStr and IV delivery of AAV5 and AAV9-PhP.B were compared for transduction and transgene expression in the cortex and striatum.

\section{MATERIALS AND METHODS}

\section{Vector Design and Production}

The AAV9-PhP.B transfer plasmid was designed by adding the PhP.B peptide to VP1 between amino acids 588 and 589 of AAV9 adapted for baculovirus production. AAV5-PhP.B was constructed by swapping in the 13-mer loop containing PhP.B, as described in the study of Deverman et al. (2016), and publicly available as sequence KU056473, shown in Figure 1A.
Baculovirus was produced by homologous recombination with the transfer plasmids, as described earlier (Urabe et al., 2002; Bosma et al., 2018). Expression cassettes containing either GFP, Luc, or miRNA were generated correspondingly. To generate AAV, Spodoptera frugiperda (SF) SF+ cells were triple-infected with baculovirus containing capsid, expression cassette, and the replicon enzyme. At $72 \mathrm{~h}$ postinfection, cells were lysed, and the clarified lysate was purified on the ÄKTA explorer (FPLC chromatography system, GE healthcare, United Kingdom) using AVB sepharose (GE healthcare, United Kingdom). The vectors were titrated by SYBR Green for quantitative PCR (qPCR) using a primer pair binding to the promoter region CAG (forward primer: GAG CCG CAG CCA TTG C and reverse primer: CAC AGA TTT GGG ACA AAG GAA GT) or CMV (forward primer: AATGGGCGGTAGGCGTGTA and reverse primer: AGGCGATCTGACGGTTCACTAA) and expressed as genome copies per $\mathrm{ml}(\mathrm{GC} / \mathrm{ml})$.

\section{In vitro Analysis}

Of note, $1 \times 10^{5} \mathrm{CHO}$ Lec- 2 cells were seeded in a 24 -well plate and inoculated and incubated overnight. On the following day, cells were inoculated with $1 \times 10^{11} \mathrm{GC} /$ well for a multiplicity of infection (MOI) of $1 \times 10^{6}$ in triplicate for each capsid. GFP expression was visualized 3-7 days postinoculation. $\mathrm{CHO}$ Lec- 2 cells were seeded at a density of $2.5 \times 10^{4}$ cells/well in duplicate in a black 96 -well plate and inoculated in a 10 -fold serial dilution starting at MOI of $4 \times 10^{6}$ of each capsid containing a luciferase expression cassette. Plates were incubated at $37^{\circ} \mathrm{C}$ for approximately $20 \mathrm{~h}$ and subsequently analyzed. $1 \times 10^{5} \mathrm{HuH}-$ 7 cells or $5 \times 10^{4} \mathrm{CHO}$ Lec- 2 cells were seeded in triplicate in two 24-well plate and inoculated with an MOI of $1 \times 10^{5}$ incubated for $20 \mathrm{~h}$. One plate was used for luciferase analysis and the other for DNA.

Luciferase assay was carried out using the One-Glo Luciferase Assay System (E6120, Promega, Madison, United States) according to the instruction of the manufacturer. DNA was extracted from cell lysate using the AllPrep kit (QIAGEN, Germany) according to the instruction of the manufacturer. Vector DNA was detected by qPCR using SYBR Green primers binding to the CMV promoter (forward primer: AAT GGG CGG TAG GCG TGTA and reverse primer: CAC AGA TTT GGG ACA AAG GAA GT).

\section{Animal Studies}

All animal studies described were approved by the local ethics committee for animal experimentation. Female, young adult C57Bl/6 mice (Janvier Labs, France) were used for all experiments. For IV administration, $8 \mu \mathrm{l} / \mathrm{g}$ body weight of each viral vector preparation was injected into the tail vein. For IStr administration, animals were anesthetized with Hypnorm/Dormicum, and an incision was made in the skin of the head. A small hole was drilled in the skull, and the striatum was stereotactically approached. A $2 \mu$ l of vehicle or AAV-miRNA was injected into the striatum in both hemispheres (Anteroposterior $=+0.8 \mathrm{~mm}$; mediolateral $= \pm 1.8 \mathrm{~mm}$; Dorsal/Ventral $=-3.0 \mathrm{~mm})$ using a $30-\mathrm{Ga}$ needle $(\mathrm{BD}$, Becton, Dickinson and Company, NJ, United States) and attached by 
A

AAV5 N N Q S S T A P - . . . . T G AAV5-PhP.B N N Q A Q T L A V P F K A Q A Q T G AAV9-PhP.B N H Q A Q T L A V P F K A Q A Q T G AAV9 $N$ HQA Q . . . . A Q A Q T G

B

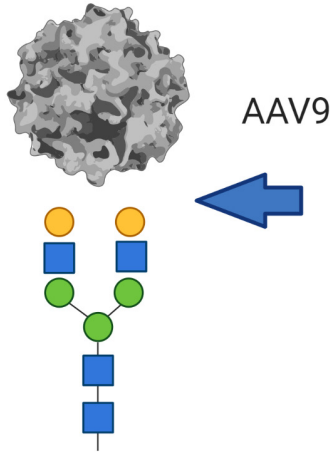

Asn N-linked

CHO Lec- 2 cells

c
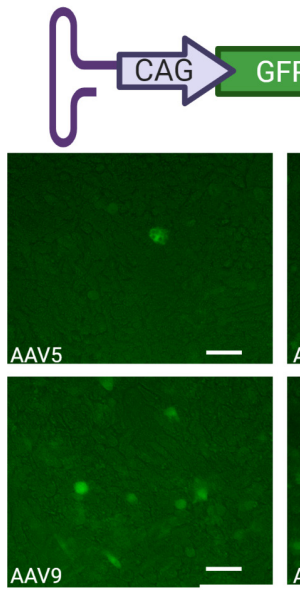

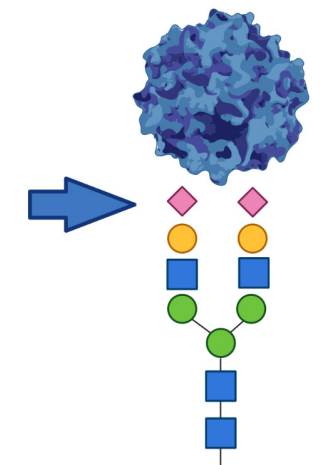

Asn N-linked
HuH-7 cells

D

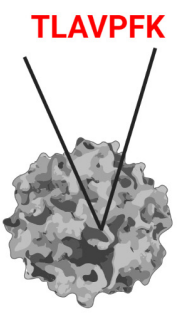

AAV9-PhP.B
AQTLAVPFKAQAQ

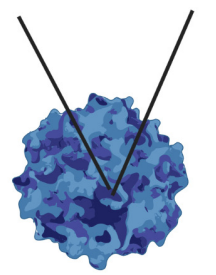

AAV5-PhP.B

AAV5

\begin{tabular}{ll|} 
Sialic acid \\
Galactose \\
Manose \\
N-acetylgalactosamine
\end{tabular}
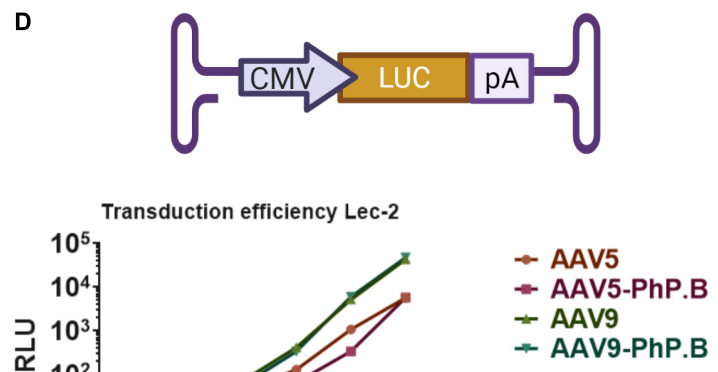

E

Transduction efficiency HuH-7 cells $1 \times 10^{5} \mathrm{MOI}$

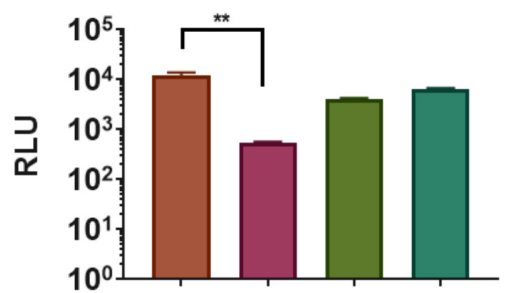

AAV5

AAV5.PhP.B

AAV9

AAV9.PhP.B

FIGURE 1 | Overview of AAV-PhP.B capsids and in vitro testing. (A) Alignment of AAV5, AAV5-PhP.B, AAV9, and AAV9-PhP.B at PhP.B insertion. (B) Binding by AAV9 of CHO Lec-2 cells due to the modification of the cell surface in glycan receptor exposing galactose, whereas AAV- 5 transduces Huh-7 cells through binding to sialic acid. Receptors on the cell surface are depicted in this figure. (C) Schematic overview of the expression cassette containing the cytomegalovirus (CMV) early enhancer element and chicken beta-actin promoter (CAG), green fluorescent protein (GFP), and poly(A) tail. CHO Lec-2 cells were inoculated in triplicate with GFP encoding constructs of each capsid. All capsids are capable of inducing GFP expression at 7 days postinoculation. (D) Graphical representation of expression cassette used containing CMV-promoter-luciferase gene and poly(A) tail. A dose-response of each capsid in CHO Lec-2 cells measured as relative luminescence (RLU). A total of $2 \times 10^{4}$ cells/well were infected in a serial dilution starting at a multiplicity of infection (MOI) of $4 \times 10^{6}$ cells/well in a 96-well plate. (E) Inoculation of $\mathrm{HuH}-7$ cells with AAV vectors. The addition of PhP.B to AAV-5 resulted in a decreased transduction in both cell types. Experiments were performed in triplicate in a 24-well plate, seeding $1 \times 10^{5}$ cells/well for HuH-7 cells. AAV5, orange bar; AAV5-PhP.B, pink bar; AAV9, light green; and AAV9-PhP.B, dark green. Asterisks indicate a statistical significance of $p \leq 0.005$. 
TABLE 1 | Overview of injection routes, vectors, and dosage used.

\begin{tabular}{|c|c|c|c|c|c|}
\hline Study & Vectors & Injection route & Volume $(\mu \mathrm{l})$ & Total dose (GC/mouse) & Titer (GC/ml) \\
\hline $\begin{array}{l}\text { Comparison of capsids at a low } \\
\text { dose }\end{array}$ & $\begin{array}{c}\text { AAV5-GFP } \\
\text { AAV5-PhP.B-GFP } \\
\text { AAV9-GFP } \\
\text { AAV9-PhP.B-GFP }\end{array}$ & IV & $200 / 25 \mathrm{gr}$ & $2,5 \times 10^{11}$ & $1 \times 10^{12}$ \\
\hline AAV9-PhP.B transduction of CNS & AAV9-PhP.B-GFP & IV & $200 / 25 \mathrm{gr}$ & $4 \times 10^{12}$ & $1,6 \times 10^{13}$ \\
\hline AAV5 vs. AAV9-PhP.B & $\begin{array}{l}\text { AAV5-miRNA } \\
\text { AAV9-miRNA }\end{array}$ & $\begin{array}{l}\text { IV } \\
\text { IStr }\end{array}$ & $\begin{array}{c}\text { 160/20 } \text { gr }^{\star} \\
4 \text { (2/hemisphere) }\end{array}$ & $\begin{array}{l}4 \times 10^{12} \\
1 \times 10^{11}\end{array}$ & $\begin{array}{l}2,7 \times 10^{13} \\
2,7 \times 10^{13}\end{array}$ \\
\hline
\end{tabular}

*Mice were 6 weeks old at the start of the experiment.

tubing to a $10-\mu 1$ Hamilton syringe at a rate of $0.5 \mu \mathrm{l} / \mathrm{min}$. After surgery, mice were administered buprenorphine (Temgesic) as pain relief. An overview of injection routes, vectors, and dosage used is given in Table $\mathbf{1}$.

\section{Vector Distribution and GFP Expression}

At 4 or 6 weeks postinjection, animals were humanely euthanized, and organs were dissected and frozen at $-80^{\circ} \mathrm{C}$. For the first experiment, in comparison with capsids at a low dose, the left hemisphere was used for molecular analysis and the other half fixed in $4 \%$ paraformaldehyde for histology. For the last experiment, both left and right hemispheres were frozen for the vector distribution analysis.

Organ pieces were pulverized to a powder using the cryoPREP system (Covaris, Woburn, MA, United States). Approximately \pm 10 -mg powder was used to extract DNA using the DNeasy ${ }^{\circledR} 96$ Blood and Tissue kit (QIAGEN, Germany). The presence of vector DNA was detected and quantified by qPCR using TaqMan primers and probe binding to CAG promoter (forward primer: GAG CCG CAG CCA TTG C, reverse primer: CAC AGA TTT GGG ACA AAG GAA GT, and probe: ATG GTA ATC GTG CGA GAG GGC GC). Vector DNA genome copies per $\mu \mathrm{g}$ DNA were quantified by interpolating from a standard line prepared from the plasmid of the expression cassette. RNA was isolated from powder using the RNeasy ${ }^{\circledR}$ Plus Mini Kit (74136) from QIAGEN, Germany. Total RNA was reverse-transcribed to cDNA using the Maxima First-Strand kit (R1362, Thermo Fisher, Waltham, MA, United States), RNA expression was quantified by using primers binding to GFP (forward primer: AGC AAA GAC CCC AAC GAG AA, reverse primer: GCG GCG GTC ACG AAC TC, and probe: CGC GAT CAC ATG GTC CTG CT) and GAPDH (TaqMan expression array from Thermo Fisher, Paisley, United Kingdom) as a housekeeping gene for reference. RNA expression was calculated using the $\triangle \mathrm{CT}$ method normalized to the housekeeping gene.

\section{microRNA TaqMan Assay}

For the detection of the expressed microRNA, pulverized cortical and striatal tissue was subjected to the Direct-zol kit (R2061, ZYMO Research, Irvine United States) according to the protocol of the manufacturer. RNA was reverse-transcribed into cDNA using the TaqMan ${ }^{\circledR}$ MicroRNA Reverse Transcription Kit (Applied Biosystems, 4366597, Waltham, MA, United States). Diluted samples of each cDNA sample were subjected to TaqMan
qPCR using a primer-probe set specific for miRNA and an internal control miRNA (U6).

\section{Graphs, Statistical Analysis, and Figures}

The statistical analysis and graphs were generated using GraphPad, www.graphpad.com [GraphPad Prism version 8.4.2. (679) for Windows, GraphPad Software, San Diego, CA, United States]. To compare groups, a one-way ANOVA was used, followed by the Tukey's multiple comparisons test. Figures with cartoons were created using biorender.com. Graphical Abstract was made by Sabela DeScience.

\section{Histology}

Of note, 24-48 h after immersion fixation in $4 \%$ paraformaldehyde, brains were embedded in $10 \%$ gelatin (Difco) in a phosphate-buffered saline (PBS). Embedded tissue was sectioned on a Vibratome. Coronal sections were collected in PBS in series at a thickness of $50 \mu \mathrm{M}$. Immunohistochemistry (IHC) was performed on the free-floating sections. Endogenous peroxidase block was performed by incubating sections for $1 \mathrm{~h}$ in $1 \% \mathrm{H}_{2} \mathrm{O}_{2} / 30 \%$ ethanol in PBS. Sections were washed three times with washing buffer (PBS/0.05\% Tween). Nonspecific blocking was performed by incubating sections for $1 \mathrm{~h}$ in PBS supplemented with $4 \%$ bovine serum albumin (BSA) and 5\% normal goat serum (NGS). Subsequently, sections were incubated overnight with primary antibody against GFP (Abcam ab290, Cambridge, United Kingdom), diluted 1:1000 in PBS/1\% BSA/1.25\% NGS/0.5\% Tween, and incubated for $1 \mathrm{~h}$ with horseradish peroxidase (HRP)-conjugated anti-rabbit before detection with 3,3'-diaminobenzidine (DAB) according to instructions of the manufacturer (Dako EnVision kit K4009, Agilent, Santa Clara, CA, United States). Subsequently, they were dehydrated through ethanol series, xylene, and embedded in Entellan before microscopic analysis.

\section{RESULTS}

\section{Design and in vitro Testing of AAV-PhP.B Capsids}

To ensure that the vectors could be adapted to our production system, PhP.B was added to AAVs adapted for the baculovirus expression system. For AAV9, PhP.B was added at the same position, I-588, as described in the study of Deverman et al. (2016). 
Earlier attempts to modify AAV5 at the corresponding position I575 were not successful (Khabou et al., 2016). An alignment of AAV5 and AAV9-PhP.B shows sequence similarity outside of the known variable regions, forming a loop at the 3-fold axis of symmetry (Figure 1A). This loop facilitates interaction with the receptors of targeted cells. To add this loop to AAV5, AAV5-PhP.B (i.e., AQTLAVPFKAQAQ) was made by swapping in the flaking regions of AAV9-PhP.B containing the peptide (Figure 1A).

The new capsids were tested for transduction efficacy in vitro using Chinese hamster ovary ( $\mathrm{CHO}$ ) Lec- 2 cells and $\mathrm{HuH}$ 7 cells. Having an easily accessible nonmurine cell line is essential to set up future potency experiments. CHO Lec2 cells are deficient in monophosphate-SA (CMP-SA) Golgi transporter. Therefore, $\mathrm{N}$ - and $\mathrm{O}$-glycans of these cells only contain galactose residues, as depicted in Figure 1B. CHO Lec2 cells are suitable for studying AAV9 transduction in vitro as this virus uses galactose residues to enter the cell (Bell et al., 2012). HuH-7 cells were used to study AAV5 preferential in vitro transduction by sialic acid residues. $\mathrm{HuH}-7$ cells are human differentiated hepatocytes derived from a cellular carcinoma cell line (Figure 1B).

The CHO Lec-2 cells were inoculated with each capsid at an MOI of $1 \times 10^{6} \mathrm{GC} /$ cell using GFP as a transgene and monitored over time to assess the ability of capsids to express a transgene. All capsids showed GFP expression 7 days after inoculation (Figure 1C). To verify the potency of AAVPhP.B, CHO Lec-2 cells were transduced with each capsid in a 10 -fold serial dilution, starting with an MOI of $4 \times 10^{6}$ GC/cell. A dose-response was observed for each capsid, showing the potency of the capsids (Figure 1D). No difference was found in the transduction efficiency of AAV9-PhP.B vs. AA9 (Figure 1D). As expected, the potency of AAV5 and AAV5PhP.B was lower. Surprisingly, in $\mathrm{HuH7}$ cells, the addition of
PhP.B to AAV5 resulted in a significant reduction in transgene activity (Figure 1E).

\section{At a Low Dose, AAV9-PhP.B Can Cross the BBB to Reach the Mouse Brain}

AAV5, AAV5-PhP.B, AAV9, and AAV9-PhP.B were evaluated in vivo in $\mathrm{C} 57 \mathrm{Bl} / 6$ mice for their capacity to cross the $\mathrm{BBB}$ following IV injection and a life period of 6 weeks. Mice were administered a relatively low dose $\left(2.5 \times 10^{11} \mathrm{GC} /\right.$ mouse $)$ of each capsid containing GFP as transgene (Figure 2A). Vector distribution was assessed in the cortex, striatum, and thalamus by qPCR. Only AAV9-PhP.B showed significant transduction to all three investigated brain areas (Figure 2B). However, the numbers of vector copies were very low to generate a positive GFP staining on IHC sections. The addition of the PhP.B peptide to AAV5 did not alter the ability of this capsid to cross the BBB (Figure 2B).

\section{AAV5-PhP.B Shows Increased GFP mRNA Expression in Mice Liver}

In vitro experiments showed a reduction in transduction and luciferase expression in AAV5-PhP.B-transduced $\mathrm{HuH}-7$ cells compared with AAV5 and no difference between AAV9 and AAV9-PhP.B. We also assessed the transduction of peripheral organs in vivo and liver GFP mRNA expression in mice. A trend is observed in the higher levels of AAV9 and AAV9PhP.B vector in the spleen. In the kidney, most of the vector is found in animals administered with AAV9-PhP.B. For the liver, there appears to be an equal distribution with the least amount of vector DNA retrieved from mice administered with AAV9-PhP.B-GFP (Figure 3A). GFP mRNA expression was significantly higher in animals administered with AAV5-PhP.B and AAV9 when compared to mice administered with AAV5 or AAV9-PhP.B (Figure 3B).
A

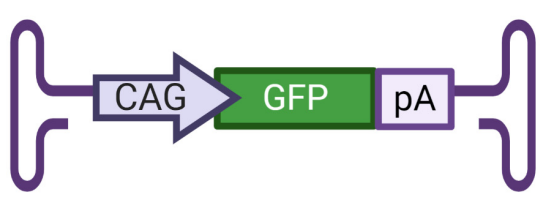

IV: $2,5 \times 10^{11} \mathrm{gc} / \mathrm{mouse}$

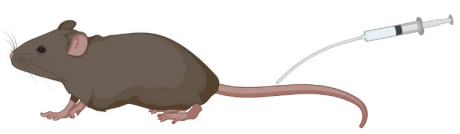

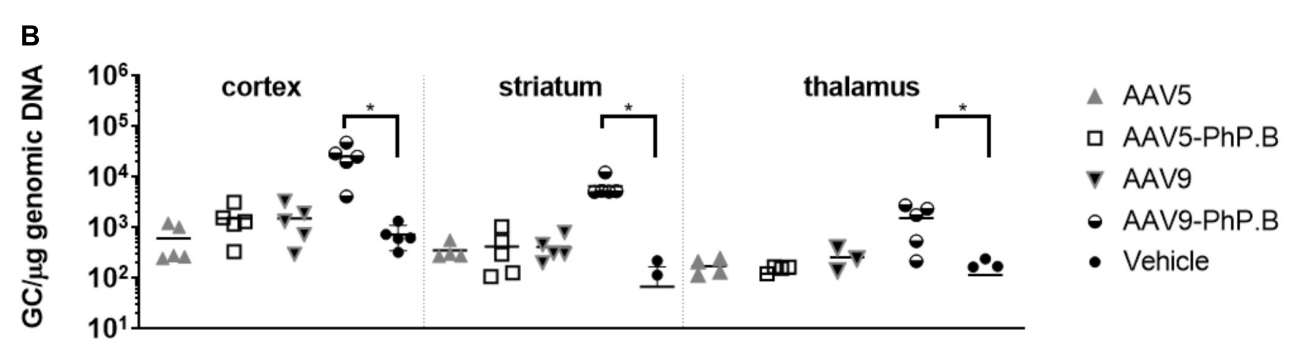

FIGURE 2 | Vector distribution in brain regions of AAV-PhP.B-GFP-administered mice. (A) Mice $(n=5)$ were administered $2.5 \times 10^{11}$ GC/mouse by intravenous (IV) injection. The expression cassette used contained the CAG, GFP, and poly(A) tail. (B) Tissue was collected 6 weeks after surgery and analyzed for the distribution of the vectors. Significantly more vector DNA was retrieved from the cortex, striatum, and thalamus of AAV-PhP.B-GFP-administered mice when compared to vehicle (PBS). The statistical analysis was performed by a one-way ANOVA followed by the Tukey's multiple comparisons test, comparing groups with vehicle. Asterisks indicate a statistical significance of $p \leq 0.05$. 


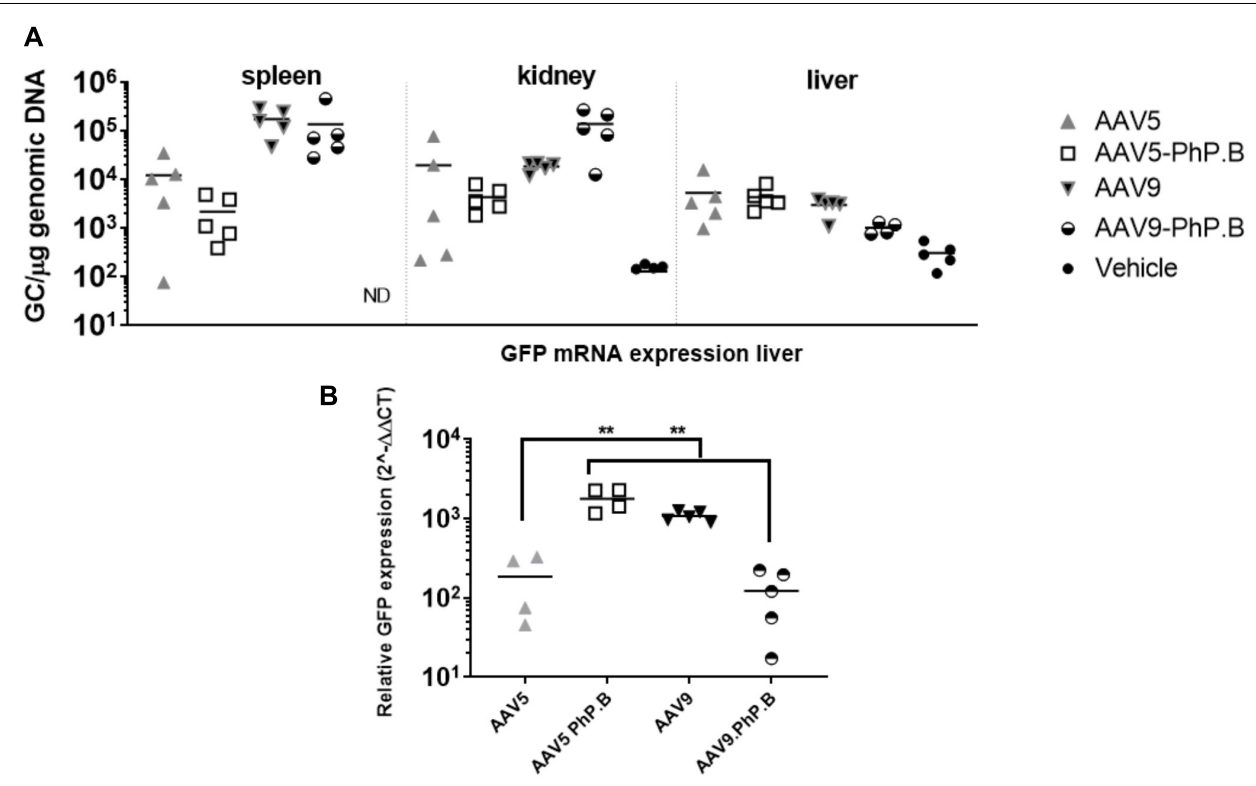

FIGURE 3 | Vector distribution in mice periphery after IV administration of AAV and liver expression. (A) Vector distribution in the spleen, kidney, and liver. (B) GFP mRNA expression in the liver with elevated levels in AAV5-PhP.B compared with AAV5. Asterisks indicate a statistical significance of $p \leq 0.005$.

\section{Central Nervous System Transduction of AAV9-PhP.B}

Genomic copies of AAV were detected and quantified after IV administration of AAV9-PhP.B. However, no GFP expression was observed in brain sections (data not shown) using the relatively low viral dose. To assess the extent of the AAV9-PhP.B-mediated transduction, GFP-transgene-carrying AAV9-PhP.B was administered at a relatively high dose $\left(4 \times 10^{12}\right.$ $\mathrm{GC} /$ mouse) to demonstrate the presence of transgene protein within the range of detection (Figure 4A). GFP expression was observed throughout the brain from the medulla to the frontal cortex. Positive neurons and astrocytes were identified by shape and location in the medulla (Figure 4B), the locus coeruleus (Figure 4C), the thalamus (Figure 4D), the hippocampus (Figure 4E), the striatum (Figure 4F), and the cortex (Figure 4G). These results highlight the potential of AAV9-PhP.B to express transgene throughout the CNS.

\section{Intrastriatal Delivery of AAV5-miRNA Results in High Distribution and Levels of microRNA in Mouse Brain}

After establishing that AAV9-PhP.B delivers transgene throughout the mouse brain, we assessed if AAV9-PhP.B could be used for predominantly adult-onset diseases such as $\mathrm{HD}$ or Parkinson's disease. For HD, various huntingtin-geneexpression-lowering strategies are in development (Miniarikova et al., 2018). At the moment, in preclinical studies, IStr administration is the method applied to reach the areas affected in HD. This experiment also aimed to evaluate if AAV9-PhP.B administered by IV could be used as a noninvasive alternative to deliver miRNA in mice.
AAV5 or AAV9-PhP.B containing a miRNA expression cassette (Figure 5A) was produced and administered IStr $\left(1 \times 10^{11} \mathrm{GC} /\right.$ mouse $)$ or IV $\left(4 \times 10^{12} \mathrm{GC} /\right.$ mouse $)$ at an equal concentration $\left(2.7 \times 10^{13} \mathrm{GC} / \mathrm{ml}\right)$, adjusting the volume to the method used (Figure 5B). This is the highest dose that could be produced without the possible formation of aggregates. No significant difference was observed when AAV9-PhP.B was administered IV or IStr. Significantly higher vector genome levels were retrieved from the striatum (Figure 5C) and the cortex (Figure 5D) of mice administered with AAV5-miRNA IStr compared with the other experimental groups. When compared to an IV administration of AAV9-PhP.B, a 22-fold and 15-fold higher vector distribution was achieved in the striatum and cortex, respectively. The levels of miRNA following AAV had a similar pattern as vector DNA found in the striatum (Figure 5E) and the cortex (Figure 5F).

\section{DISCUSSION}

In this study, AAV9-PhP.B and AAV5-PhP.B were produced in the baculovirus expression system and showed to transduce cells in vitro. Subsequently, these constructs were evaluated in vivo. Although the addition of PhP.B does not enhance BBB crossing of serotype GFP-carrying AAV5, it does increase GFP mRNA expression in the liver. We found that the addition of PhP.B to AAV9 enhances the capability of the vector to cross the BBB, as found in the earlier studies (Deverman et al., 2016; Morabito et al., 2017; Hordeaux et al., 2018), and, when applied at a sufficient dose, GFP expression can be noted throughout the brain from the medulla to the cortex. IStr and IV administration of AAV9-PhP.B mediated similar transduction levels in the cortex and striatum. When we compared IStr administration of AAV5 to 
A

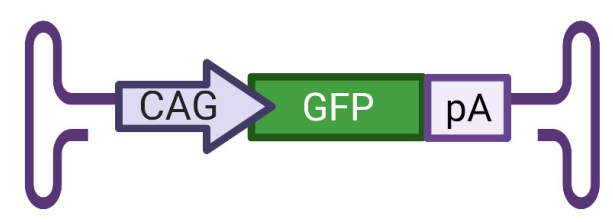

B

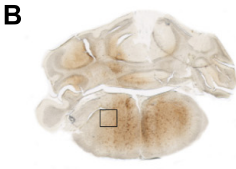

C

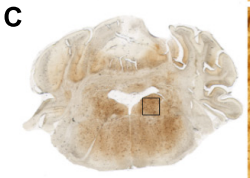

D

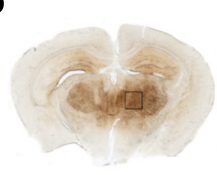

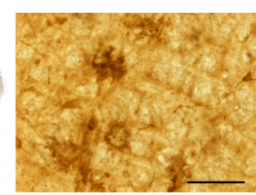
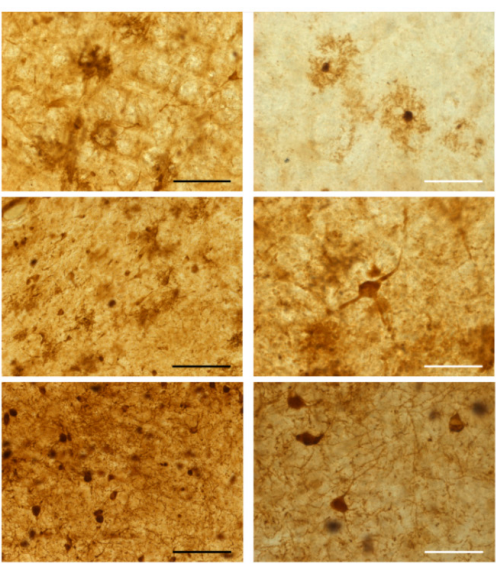

\section{IV: $4 \times 10^{12} \mathrm{gc} /$ mouse}

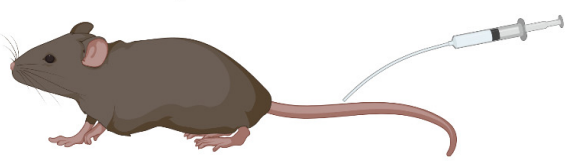

E

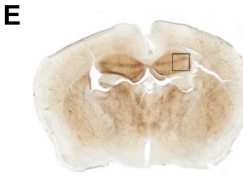

$\mathbf{F}$
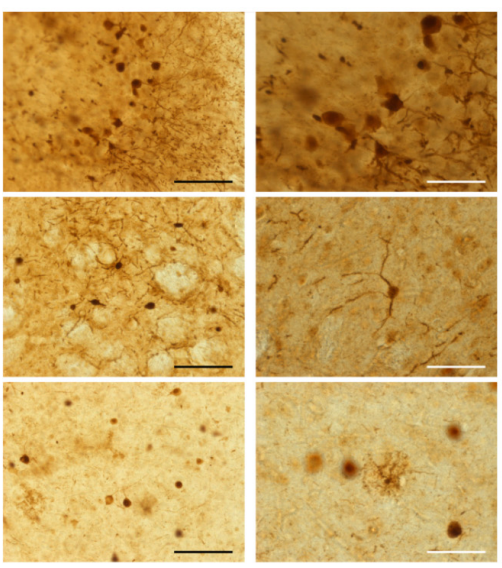

FIGURE 4 | GFP expression throughout the brain of mice administered with AAV9-PhP.B. (A) Cartoon depicting construct used, administration route, and dose used of $4 \times 10^{12} \mathrm{GC} /$ mouse. Brains were extracted, fixed in $4 \%$ paraformaldehyde, embedded in gelatin, and serially cut by using a Vibratome to $50-\mu \mathrm{m}$ sections. GFP protein staining (shown in brown) in (B) the medulla, (C) the locus coeruleus, (D) the thalamus, (E) the hippocampus, (F) the striatum, and (G) the cortex. Rectangles show the location of the micrograph taken. The black scale bar represents $50 \mu \mathrm{m}$, and white bar represents $250 \mu \mathrm{m}$.

IV administration of AAV9, AAV5 IStr was superior in terms of transducing the cortex and striatum.

Overall, we observed lower transduction levels with AAV9 vectors using a similar dose as described in the study first describing PhP.B (Deverman et al., 2016). The difference in the quantification method per laboratory could be a possible explanation for observed differences. In addition, for AAV9, varying results are reported between laboratories with regard to the distribution patterns. For example, in one of the first studies, predominant astrocyte transduction (Foust et al., 2008) was reported while another laboratory observed the transduction of neurons (Gray et al., 2011). Another observation is more variation in the group of animals administered IStr compared with the IV-injected group. Vector spread after the striatal infusion is not homogenous throughout the tissue since this is performed with concentrated vector directly into tissue resulting in local differences. Therefore, more variation is observed between mice. We suspected that transfer of a homogenous dilution in blood from the endothelium to the striatal area occurs in a more homogenous manner and is, therefore, less sensitive to variation between animals.

The addition of PhP.B to AAV5 resulted in increased transgene mRNA in the liver compared with AAV5. Ly6a is constitutively expressed by liver sinusoidal endothelial cells (LSEC) (Luna et al., 2004). The LSEC form a permeable barrier between the bloodstream and hepatocytes (Poisson et al., 2017). In the brain, the interaction between AAV9-PhP.B and Ly6A facilitates the crossing of the BBB. Since we did not note the crossing of the BBB by AAV5-PhP.B, the LSEC may be transduced rather than the hepatocytes underneath.
A recent publication (Powell et al., 2020) showed that AAV9 after infection could mediate expression in different cell types in the brain, depending on the promoter used. Moreover, a six alanine insertion after the VP2 start residue results in a shift from oligodendrocyte to neuronal transduction. The addition of PhP.B to AAV could also have an effect on the specific cells transduced within the tissue. Recently, RNAscope in situ hybridization revealed the precise location of the vector DNA and mRNA produced, while identifying specific cells by IHC (Zhao et al., 2020). This method could be applied in the future to investigate and differentiate between level of vector entry and transgene expression in a specific cell. In our case, this would be a method to investigate the liver transduction by AAV5-PhP.B. Since this method is beyond the scope of this study, we did not pursue this further.

The question remains why AAV9 is enhanced by PhP.B to cross the BBB, while AAV5 is not. The mechanism by which AAV crosses the BBB has not been fully understood. AAV9 appears to cross the $\mathrm{BBB}$ by transcytosis in an in vitro human BBB model by a yet unknown receptor (Merkel et al., 2017). The data in mice indicated that AAV9-PhP.B crosses the BBB by transcytosis of brain microvascular endothelial cells (Matsuzaki et al., 2019). The results of this study with AAV5-PhP.B indicate that PhP.B alone is not enough for an AAV particle to cross the endothelial barrier. Therefore, it appears PhP.B facilitates the transduction of endothelial cells which in turn enables the enhanced trafficking of AAV9 through the endothelium from apical to basolateral side by another co-receptor. For AAV5PhP.B endocytosis and trandusction of the endothelium might 
A

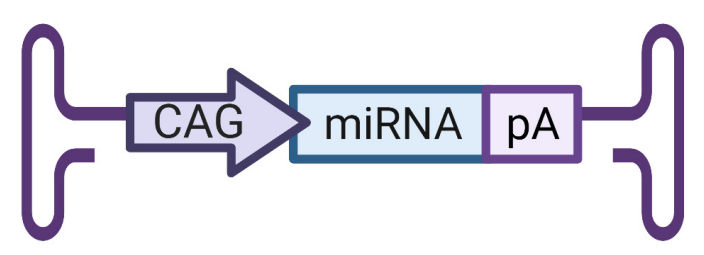

B

IV:

$4 \times 10^{12} \mathrm{gc} /$ mouse

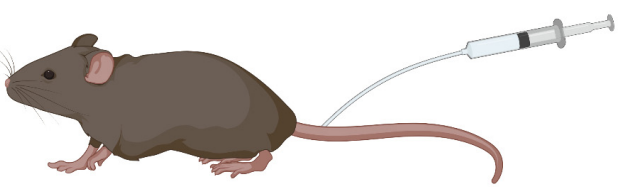

c

striatum

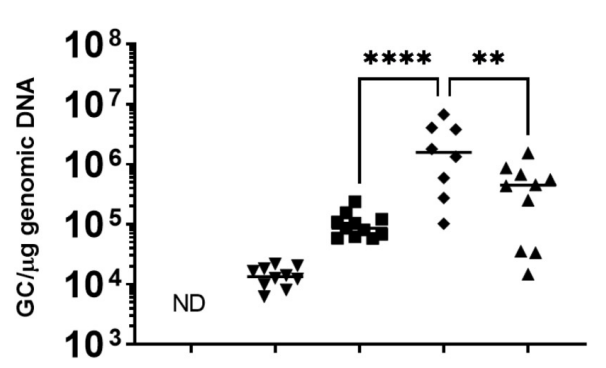

E

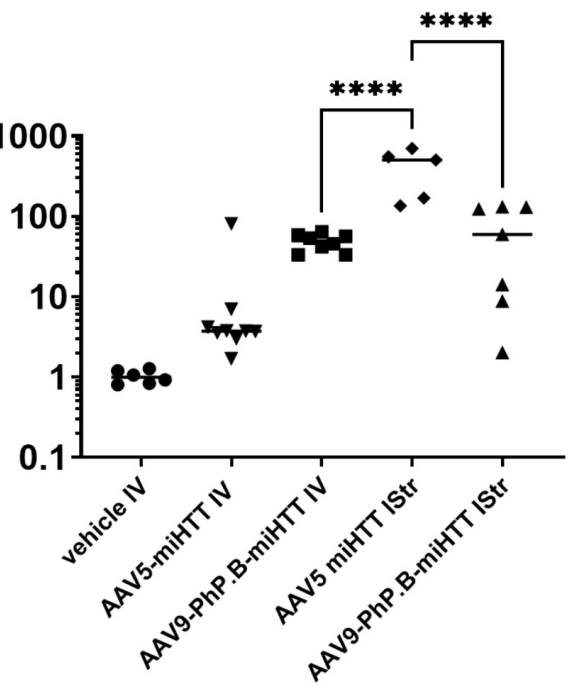

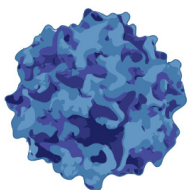

AAV5

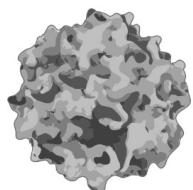

AAV9-PhP.B

IStr

$1 \times 10^{11} \mathrm{gc} /$ mouse

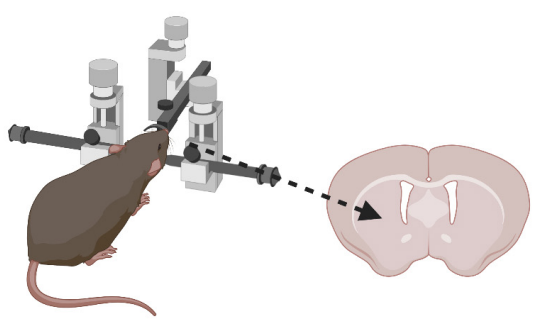

D

cortex

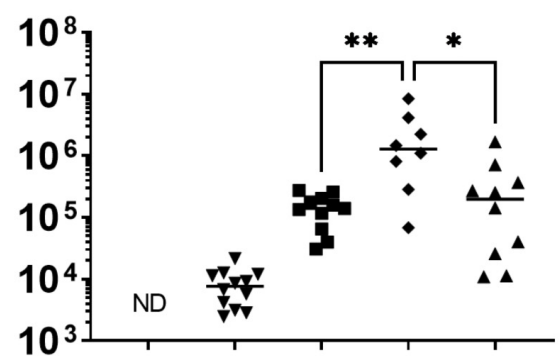

$\mathbf{F}$

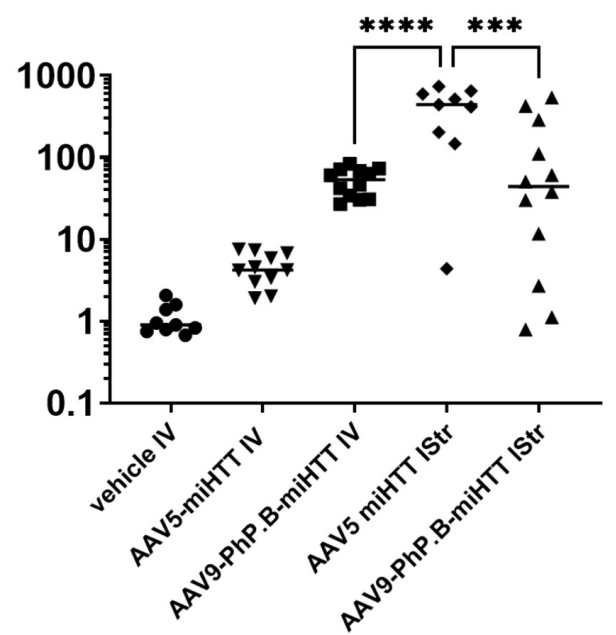

FIGURE $\mathbf{5}$ | Vector distribution and miRNA expression in mice brain after IV or intrastriatal (IStr) delivery. (A) A graphical representation of vectors used in this study. The expression cassette used consisted of CAG-promoter-miRNA and poly(A) tail. (B) Administration routes at which mice were either administered intravenously (IV) or IStr. (C) Distribution in the striatum. A significantly higher amount of vector DNA was observed in the striatum and cortex of mice that received AAV5 IStr. For AAV5, direct administration results in significantly higher distribution, while for AAV9-PhP.B, similar amounts of the vector were retrieved on average. (D) Distribution in the cortex. In the cortex, the same pattern is observed as in the striatum. The IStr administration of AAV5 results in higher amounts of vector DNA than the IV administration of AAV9-PhP.B or AAV5 IV. (E) miRNA levels in the striatum and (F) miRNA levels in cortex. The vector DNA results translate to miRNA expression levels. In this study, we also observed higher miRNA levels in mice administered AAV5 IStr compared with AAV9-PhP.B IV and AAV5-PhP.B. ND stands for not detected. Statistical analysis was performed by the one-way ANOVA, followed by the Bonferroni multiple comparisons test. Asterisks indicate a statistical significance of $* p \leq 0.05,{ }^{* *} p<0.005,{ }^{* * *} p \leq 0.001$, and ${ }^{* * * *} p<0.0001$. 
still occur but the astrocytes and neurons underneath remain untransduced.

The addition of different peptides to the corresponding position 1587 in AAV2 enhanced the AAV2 ability to transduce the CNS after IV administration (Chen et al., 2009). Therefore, it is theoretically possible to add a peptide to an AAV serotype other than AAV9 and increase BBB crossing. Each of the serotypes uses a different primary receptor. AAV2 binds to heparin sulfate proteoglycan (HSPG), AAV9 to N-linked galactose receptors, and AAV5 to N-linked sialic acid (Vance et al., 2015). However, both AAV2 and AAV9 use laminin receptor (LamR) as a secondary receptor, while AAV5 also binds the platelet-derived growth factor receptor (PDGF-R) (Vance et al., 2015). The interplay between these various receptors appears to play a role in various transduction patterns.

The AAV9-based IV therapy in combination with a PhP.B designed for primate could be a viable option for diseases with multifocal pathologies where different types of cells in the body are affected, such as lysosomal storage diseases (Tardieu et al., 2017). In most cases, ideally, therapy should be applied as early as possible before irreversible neurodegeneration occurs (Massaro et al., 2020). Due to the more permissiveness of the BBB, newborn babies need a lower dose for disease correction, implying a lower risk of the therapy. In diseases where the whole body is genetically affected, the systemic mechanism of AAV9-primate PhP.B might even be an advantage.

For a clinical application, a primate permissive version of PhP.B should be developed. A putative human equivalent of murine Ly6a, the receptor to which PhP.B binds, is Ly6E. The Ly6E protein is highly expressed in human $\mathrm{BBB}$ endothelial cells. Furthermore, a peptide sequence within HIV-GP120 is hypothesized as a potential binding motif functioning as a primate permissive PhP.B (Ille et al., 2020). This peptide could be used to develop a human variant of AAV9-PhP.B to be tested in nonhuman primates or in vitro systems that mimic the human $\mathrm{BBB}$.

Our results further show that the direct administration of AAV5 represents an attractive strategy for diseases where the deeper brain areas have to be targeted. AAV5 has been studied for localized administration to various CNS targets such as intrathalamic pathway (Samaranch et al., 2017) in the deep cerebellar nuclei (Martier et al., 2019b) and IStr. The IStr administration of AAV5 has been extensively studied in multiple models. Our current transduction results are in line with those of a rodent preclinical HD model in which disease amelioration was achieved (Spronck et al., 2019). The IStr administration of AAV5 has also been studied in nonhuman primates (Samaranch et al., 2017) and minipigs (Evers et al., 2018). A similar transduction pattern is observed across all studied species, with high transduction and transgene expression levels in the striatum

\section{REFERENCES}

Bell, C. L., Gurda, B. L., Vliet, K., Agbandje-McKenna, M., and Wilson, J. M. (2012). Identification of the galactose binding domain of the adeno-associated virus serotype 9 capsid. J. Virol. 86, 7326-7333. doi: 10.1128/JVI.00448-412

Bosma, B., du Plessis, F., Ehlert, E., Nijmeijer, B., de Haan, M., Petry, H., et al. (2018). Optimization of viral protein ratios for production of rAAV serotype 5 and cortex. A clinical study using the IStr administration of AAV5 has been initiated in patients with HD (NCT04120493).

We studied the transduction and miRNA expression in the cortex and striatum following AAV delivery. Irrespective of the delivery method, similar transduction and transgene expression levels are observed in the brain with AAV9-PhP.B, showing that for an AAV9 vector with PhP.B or similar enhancement, there would be no added benefit of an IStr over IV delivery. From the standpoint of a clinical program targeting solely the striatum and the connection areas, it is worth investing in IStr delivery with AAV5 rather than further optimizing AAV9 or another capsid to reach the striatum after IV delivery.

Taken together, our results show that for therapies where high levels of transduction are needed, the direct delivery of AAV5 is a viable option. For treatments in which a wide viral spreading throughout the brain and body is desired, a human-targeting variant of AAV9-PhP.B is an attractive candidate.

\section{DATA AVAILABILITY STATEMENT}

The raw data supporting the conclusions of this article will be made available by the authors, without undue reservation upon request.

\section{ETHICS STATEMENT}

The animal study was reviewed and approved by Animal Welfare Body AMC.

\section{AUTHOR CONTRIBUTIONS}

$\mathrm{KP}$ and $\mathrm{BB}$ : conceptualization and initial draft. KP, FP, SP, JL, and $\mathrm{BB}$ : investigation. SD, PK, GM, and BB: supervision and editing. All authors contributed to the article and approved the submitted version.

\section{FUNDING}

This study was financially supported by uniQure.

\section{ACKNOWLEDGMENTS}

We would like to thank S. Baatje, C. Brouwers, L. Paerels, I. Hinojo, and the vector development group for their technical assistance. We would also like to thank E. Broug, R. Martier, and E. Sawyer for critically reviewing this manuscript.

in the baculovirus system. Gene Ther. 25, 415-424. doi: 10.1038/s41434-0180034-37

Caron, N. S., Southwell, A. L., Brouwers, C. C., Cengio, L. D., Xie, Y., Black, H. F., et al. (2019). Potent and sustained huntingtin lowering via AAV5 encoding miRNA preserves striatal volume and cognitive function in a humanized mouse model of Huntington disease. Nucleic Acids Res. 48, 36-54. doi: 10.1093/nar/ gkz976 
Chen, W., Hu, Y., and Ju, D. (2020). Gene therapy for neurodegenerative disorders: advances, insights and prospects. Acta Pharm. Sinica B 10, 1347-1359. doi: 10.1016/j.apsb.2020.01.015

Chen, Y. H., Chang, M., and Davidson, B. L. (2009). Molecular signatures of disease brain endothelia provide new sites for CNS-directed enzyme therapy. Nat. Med. 15, 1215-1218. doi: 10.1038/nm.2025

Deverman, B. E., Pravdo, P. L., Simpson, B. P., Kumar, S., Chan, K. Y., Banerjee, A., et al. (2016). Cre-dependent selection yields AAV variants for widespread gene transfer to the adult brain. Nat. Biotechnol. 34, 204-209. doi: 10.1038/nbt. 3440

Emborg, M. E., Hurley, S. A., Joers, V., Tromp, D. P. M., Swanson, C. R., OhshimaHosoyama, S., et al. (2014). Titer and product affect the distribution of gene expression after intraputaminal convection-enhanced delivery. Stereotactic Funct. Neurosurg. 92, 182-194. doi: 10.1159/000360584

Evers, M. M., Miniarikova, J., Juhas, S., Vallès, A., Bohuslavova, B., Juhasova, J., et al. (2018). AAV5-miHTT gene therapy demonstrates broad distribution and strong human mutant huntingtin lowering in a Huntington disease minipig model. Mol. Ther. 26, 2163-2177. doi: 10.1016/j.ymthe.2018.06.021

Foust, K. D., Nurre, E., Montgomery, C. L., Hernandez, A., Chan, C. M., and Kaspar, B. K. (2008). Intravascular AAV9 preferentially targets neonatal neurons and adult astrocytes. Nat. Biotechnol. 27, 59-65. doi: 10.1038/nbt. 1515

Gray, S. J., Matagne, V., Bachaboina, L., Yadav, S., Ojeda, S. R., and Samulski, J. R. (2011). Preclinical differences of intravascular AAV9 delivery to neurons and glia: a comparative study of adult mice and nonhuman primates. Mol. Ther. 19, 1058-1069. doi: 10.1038/mt.2011.72

Hordeaux, J., Wang, Q., Katz, N., Buza, E. L., Bell, P., and Wilson, J. M. (2018). The neurotropic properties of AAV-PHP.B are limited to C57BL/6J mice. Mol. Therapy 26, 664-668. doi: 10.1016/j.ymthe.2018.01.018

Hordeaux, J., Yuan, Y., Clark, P. M., Wang, Q., Martino, A. R., Sims, J. J., et al. (2019). The GPI-linked protein LY6A (SCA-1) drives AAV-PHP.B transport across the blood-brain barrier. Mol. Therapy 27, 912-921. doi: 10.1016/j.ymthe. 2019.02.013

Huang, Q., Chan, K. Y., Tobey, I. G., Chan, Y. A., Poterba, T., Boutros, C. L., et al. (2019). Delivering genes across the blood-brain barrier: LY6A, a novel cellular receptor for AAV-PHP.B capsids. PLoS One 14:e0225206. doi: 10.1371/journal. pone.0225206

Ille, A. M., Kishel, E., Bodea, R., Ille, A., Lamont, H., and Amico-Ruvio, S. (2020). Protein LY6E as a candidate for mediating transport of adeno-associated virus across the human blood-brain barrier. J. Neurovirol. 26, 769-778. doi: 10.1007/ s13365-020-00890-899

Keskin, S., Brouwers, C. C., Sogorb-Gonzalez, M., Martier, R., Depla, J. A., Vallès, A., et al. (2019). AAV5-miHTT lowers huntingtin mRNA and protein without off-target effects in patient-derived neuronal cultures and astrocytes. Mol. Ther. Methods Clin. Dev. 15, 275-284. doi: 10.1016/j.omtm.2019.09.010

Khabou, H., Desrosiers, M., Winckler, C., Fouquet, S., Auregan, G., Bemelmans, A.-P., et al. (2016). Insight into the mechanisms of enhanced retinal transduction by the engineered AAV2 capsid variant $-7 \mathrm{~m} 8$ : mechanisms of enhanced transduction by AAV2-7m8. Biotechnol. Bioeng. 113, 2712-2724. doi: 10.1002/bit. 26031

Kotin, R. M., and Snyder, R. O. (2017). Manufacturing clinical grade recombinant adeno-associated virus using invertebrate cell lines. Hum. Gene Therapy 28, 350-360. doi: 10.1089/hum.2017.042

Liguore, W. A., Domire, J. S., Button, D., Wang, Y., Dufour, B. D., Srinivasan, S., et al. (2019). AAV-PHP.B administration results in a differential pattern of CNS biodistribution in non-human primates compared to mice. Mol. Ther. 27, 2018-2037. doi: 10.1016/j.ymthe.2019.07.017

Luna, G., Paez, J., and Cardier, J. E. (2004). Expression of the hematopoietic stem cell antigen Sca-1 (LY-6A/E) in liver sinusoidal endothelial cells: possible function of sca-1 in endothelial cells. Stem Cells Dev. 13, 528-535. doi: 10.1089/ scd.2004.13.528

Martier, R., Liefhebber, J. M., García-Osta, A., Miniarikova, J., Cuadrado-Tejedor, M., Espelosin, M., et al. (2019a). Targeting RNA-mediated toxicity in C9ORF72 ALS/FTD by RNAi-based gene therapy. Mol. Therapy - Nucleic Acids 16, 26-37. doi: 10.1016/j.omtn.2019.02.001

Martier, R., Sogorb-Gonzalez, M., Stricker-Shaver, J., Hübener-Schmid, J., Keskin, S., Klima, J., et al. (2019b). Development of an AAV-Based MicroRNA gene therapy to treat machado-joseph disease. Mol. Ther. Methods Clin. Dev. 15, 343-358. doi: 10.1016/j.omtm.2019.10.008

Massaro, G., Hughes, M. P., Whaler, S. M., Wallom, K.-L., Priestman, D. A., Platt, F. M., et al. (2020). Systemic AAV9 gene therapy using the synapsin I promoter rescues a mouse model of neuronopathic Gaucher disease but with limited cross-correction potential to astrocytes. Hum. Mol. Genet. 29, 1933-1949. doi: $10.1093 / \mathrm{hmg} / \mathrm{ddz} 317$

Matsuzaki, Y., Konno, A., Mochizuki, R., Shinohara, Y., Nitta, K., Okada, Y., et al. (2018). Intravenous administration of the adeno-associated virus-PHP.B capsid fails to upregulate transduction efficiency in the marmoset brain. Neurosci. Lett. 665, 182-188. doi: 10.1016/j.neulet.2017. 11.049

Matsuzaki, Y., Tanaka, M., Hakoda, S., Masuda, T., Miyata, R., Konno, A., et al. (2019). Neurotropic properties of AAV-PHP.B are shared among diverse inbred strains of mice. Mol. Ther. 27, 700-704. doi: 10.1016/j.ymthe.2019.02.016

Mendell, J. R., Al-Zaidy, S., Shell, R., Arnold, D. W., Rodino-Klapac, L. R., Prior, T. W., et al. (2017). Single-Dose Gene-Replacement therapy for spinal muscular atrophy. N. Engl. J. Med. 377, 1713-1722. doi: 10.1056/nejmoa170 6198

Merkel, S. F., Andrews, A. M., Lutton, E. M., Mu, D., Hudry, E., Hyman, B. T., et al. (2017). Trafficking of adeno-associated virus vectors across a model of the blood-brain barrier; a comparative study of transcytosis and transduction using primary human brain endothelial cells. J. Neurochem. 140, 216-230. doi: 10.1111/jnc.13861

Mijanović, O., Branković, A., Borovjagin, A. V., Butnaru, D. V., Bezrukov, E. A., Sukhanov, R. B., et al. (2020). Battling neurodegenerative diseases with adeno-associated virus-based approaches. Viruses 12:460. doi: 10.3390/v120 40460

Miniarikova, J., Evers, M., and Konstantinova, P. (2018). Translation of microRNAbased huntingtin lowering therapies from preclinical studies to the clinic. Mol. Ther. 26, 947-962. doi: 10.1016/j.ymthe.2018.02.002

Miniarikova, J., Zimmer, V., Martier, R., Brouwers, C. C., Pythoud, C., Richetin, K., et al. (2017). AAV5-miHTT gene therapy demonstrates suppression of mutant huntingtin aggregation and neuronal dysfunction in a rat model of Huntington's disease. Gene Ther. 24, 630-639. doi: 10.1038/gt. 2017.71

Morabito, G., Giannelli, S. G., Ordazzo, G., Bido, S., Castoldi, V., Indrigo, M., et al. (2017). AAV-PHP.B-Mediated global-scale expression in the mouse nervous system enables GBAl gene therapy for wide protection from synucleinopathy. Mol. Therapy 25, 2727-2742. doi: 10.1016/j.ymthe.2017. 08.004

Poisson, J., Lemoinne, S., Boulanger, C., Durand, F., Moreau, R., Valla, D., et al. (2017). Liver sinusoidal endothelial cells: physiology and role in liver diseases. J. Hepatol. 66, 212-227. doi: 10.1016/j.jhep.2016.07.009

Powell, S. K., Samulski, R. J., and McCown, T. J. (2020). AAV capsid-promoter interactions determine CNS cell selective gene expression in vivo. Mol. Ther. 28, 1373-1380. doi: 10.1016/j.ymthe.2020.03.007

Samaranch, L., Blits, B., Sebastian, S. W., Hadaczek, P., Bringas, J., Sudhakar, V., et al. (2017). MR-guided parenchymal delivery of adeno-associated viral vector serotype 5 in non-human primate brain. Gene Ther. 24, 253-261. doi: 10.1038/gt.2017.14

Spronck, E. A., Brouwers, C. C., Vallès, A., de Haan, M., Petry, H., van Deventer, S. J., et al. (2019). AAV5-miHTT gene therapy demonstrates sustained huntingtin lowering and functional improvement in Huntington disease mouse models. Mol. Ther. - Methods Clin. Dev. 13, 334-343. doi: 10.1016/j.omtm.2019. 03.002

Tardieu, M., Zérah, M., Gougeon, M.-L., Ausseil, J., de Bournonville, S., Husson, B., et al. (2017). Intracerebral gene therapy in children with mucopolysaccharidosis type IIIB syndrome: an uncontrolled phase $1 / 2$ clinical trial. Lancet Neurol. 16, 712-720. doi: 10.1016/s1474-4422(17)3016930162

Urabe, M., Ding, C., and Kotin, R. M. (2002). Insect cells as a factory to produce adeno-associated virus Type 2 vectors. Hum. Gene Ther. 13, 1935-1943. $10.1089 / 10430340260355347$

Vance, M. A., Mitchell, A., and Samulski, R. J. (2015). Gene Therapy - Principles and Challenges. IntechOpen: Doaa Hashad. doi: 10.5772/61988

Zhang, H., Yang, B., Mu, X., Ahmed, S., Su, Q., He, R., et al. (2011). Several rAAV vectors efficiently cross the blood-brain barrier and transduce neurons 
and astrocytes in the neonatal mouse central nervous system. Mol. Therapy 19, 1440-1448. doi: 10.1038/mt.2011.98

Zhao, J., Yue, Y., Patel, A., Wasala, L., Karp, J. F., Zhang, K., et al. (2020). High-resolution histological landscape of AAV DNA distribution in cellular compartments and tissues following intramuscular and intravenous injection. Mol. Ther. Methods Clin. Dev. 18, 856-868. doi: 10.1016/j.omtm.2020.08.006

Conflict of Interest: KP, FP, SP, SD, JL, PK, and BB were employees of uniQure at the time the data wad generated.

The remaining author declares that the research was conducted in the absence of any commercial or financial relationships that could be construed as a potential conflict of interest.
Publisher's Note: All claims expressed in this article are solely those of the authors and do not necessarily represent those of their affiliated organizations, or those of the publisher, the editors and the reviewers. Any product that may be evaluated in this article, or claim that may be made by its manufacturer, is not guaranteed or endorsed by the publisher.

Copyright (c) 2021 Pietersz, Plessis, Pouw, Liefhebber, van Deventer, Martens, Konstantinova and Blits. This is an open-access article distributed under the terms of the Creative Commons Attribution License (CC BY). The use, distribution or reproduction in other forums is permitted, provided the original author(s) and the copyright owner(s) are credited and that the original publication in this journal is cited, in accordance with accepted academic practice. No use, distribution or reproduction is permitted which does not comply with these terms. 